\title{
The path to far-IR interferometry in space: recent developments, plans, and prospects
}

\author{
David Leisawitz ${ }^{* a}$ and Stephen A. Rinehart ${ }^{\mathrm{a}}$ \\ ${ }^{a}$ NASA Goddard Space Flight Center, 8800 Greenbelt Rd., Greenbelt, MD, USA 20771-2400
}

\begin{abstract}
The far-IR astrophysics community is eager to follow up Spitzer and Herschel observations with sensitive, highresolution imaging and spectroscopy, for such measurements are needed to understand merger-driven star formation and chemical enrichment in galaxies, star and planetary system formation, and the development and prevalence of waterbearing planets. The community is united in its support for a space-based interferometry mission. Through concerted efforts worldwide, the key enabling technologies are maturing. Two balloon-borne far-IR interferometers are presently under development. This paper reviews recent technological and programmatic developments, summarizes plans, and offers a vision for space-based far-IR interferometry involving international collaboration.
\end{abstract}

Keywords: far-infrared, spatio-spectral interferometry, BETTII, SPIRIT, SPECS, FIRI, IR detectors, cryogenic optics

\section{INTRODUCTION}

The time will come for space-based far-infrared interferometry, but it hasn't yet arrived. It's the right idea because the human quest to understand our place in the cosmos - How did we get here? - depends on our probing sensitively and in fine detail developing planetary systems and distant galaxies in this wavelength region, and no alternative method is as technically feasible and affordable.

Purely practical issues will lead to delay and a data gap. In the US, the astrophysics community is struggling to agree on priorities and just beginning to awaken to the notion that a $\sim$ \$B Probe-class mission line and international collaboration would open the door to a better balanced space mission portfolio along the lines of the Great Observatory program. This is particularly important in a tough economic climate. Further, the astrophysics community has been lulled by an uninterrupted, continually improving information stream from space-based far-IR astrophysics missions lasting three decades. Present-day knowledge of the cosmos would be much poorer if we hadn't found that even early galaxies were laden with dust; that interstellar clouds cool and give birth to stars because they liberate energy through far-IR spectral lines; that stars often are surrounded by dusty debris disks, the remnants of planet formation; or that half of a typical galaxy's starlight is reprocessed by dust and emerges in the far-infrared. Dust hides developing planetary systems from even the most powerful UV/optical telescopes. When the Herschel Space Observatory runs out of cryogen in early 2013, this information stream will cease to flow, and the silence will reverberate throughout the community. We're destined to hear silence for a while, for there is no plan and no budget to launch a successor to Herschel for over a decade.

This gap will not be for lack of a coherent, stable, practical plan. The plan has been in place and advocated by the far-IR community for over a decade. In section 2 this paper we offer scientific motivation for high-resolution, sensitive spatial and spectral observations in the far-IR. Section 3 summarizes the "far-IR community plan" as it was documented a decade ago. Community consensus persisted and the plan was only slightly revised for the Astro2010 Decadal Survey.

Section 4 could be titled "getting real." It describes the landscape of the real world and technical and budgetary constraints, as well as technical progress made over the past decade. Section 5 looks toward the future. If the time for space-based far-IR interferometry will come, as we contend, this section explains how it might happen.

The "path" referred to in the title of this paper is an apt metaphor. A decade ago the far-IR community envisaged the optimal straight-line path leading to space-based spatio-spectral interferometry. During the past decade, the path took some turns and potholes appeared, but the destination remained fixed. Progress has been slow, and the gap between Herschel and successor missions is now inevitable, but we march undeterred down the path toward the goal.

*David.T.Leisawitz@nasa.gov; phone1301 286-0807 


\section{THE POT OF GOLD AT THE END OF THE PATH}

Where are we trying to go, and what beckons us? The far-infrared astrophysics community is drawn by some of the most compelling questions prioritized by the broader astronomical community in the US 2010 Decadal Survey, New Worlds, New Horizons, such as these: How do planetary systems form? How did 13 billion years of protogalactic mergers and star formation lead to the development of the existing population of galaxies, including the Milky Way? ${ }^{1}$ Astrophysical information critical to our ability to answer these questions is uniquely accessible with spatio-spectral far-IR space interferometers. $^{2}$

\subsection{On the importance of far-infrared astrophysics}

The far-IR is important for many reasons. Most of the energy radiated by protostars, protoplanetary systems, and the debris disks surrounding mature stars, and half or more of a galaxy's emission, is far-IR light. Dust extinction, which completely hides many of these objects at visible wavelengths, is greatly reduced in the far-IR. Further, many important diagnostic spectral lines and features are present in the far-IR. For example, the spectrum is full of $\mathrm{H}_{2} \mathrm{O}$ lines and contains water ice features at 44 and $63 \mu \mathrm{m},{ }^{4}$ and $\mathrm{Herschel}$ has revealed important variations in molecular line strength among protostars in various development phases. ${ }^{5}$ Herschel and future interferometers will study water in the gaseous and solid states to learn how water reservoirs form in planetary systems, ${ }^{6,7}$ and how the water is delivered to planets, ${ }^{8}$ enabling life. With interferometry, we will be able to probe the temperature distributions in spatially resolved protoplanetary disks and find new planets by the dust they trap in debris disks ${ }^{9}$ by mapping the far-IR continuum emission. The far-IR spectral lines of simple hydrides, such as $\mathrm{CH}$, will advance our understanding of interstellar chemistry. ${ }^{4}$ A triplet of rest frame mid-IR lines from ionized neon, and a number of $\mathrm{C}, \mathrm{N}$, and $\mathrm{O}$ fine structure lines, are very good diagnostic probes to distinguish high-redshift Active Galactic Nuclei from galaxies powered primarily by star formation. ${ }^{10,11,12}$ Many important fine structure lines, including the $\mathrm{C}^{+} 158 \mu \mathrm{m}$ line - the single strongest line in the spectrum of the Milky Way ${ }^{13}$ - appear in the far-IR spectra of extragalactic sources at redshift $z$ up to about 5 , beyond which complementary observations can be made with the Atacama Large Millimeter Array (ALMA).

Access to space enables sensitive far-IR measurements, and past and present investments in far-IR telescopes (IRAS, COBE, Spitzer, Herschel) have been richly rewarded. Research results based on analyses of data from IRAS are pervasive in three decades of scientific literature. The European Space Agency's Infrared Space Observatory (ISO) proved the value of IR spectroscopy. And far-IR observations continue to surprise: among its many accomplishments, Spitzer went above and beyond the call of duty and enabled us to characterize the atmospheres of transiting extrasolar planets. Next-generation far-IR space instruments promise to continue to elucidate poorly understood astrophysical phenomena while providing new scientific surprises:

\subsection{The case for wide-field spatio-spectral interferometry}

Herschel provides unprecedented angular resolution in the far-IR ( 7 arcsec at $100 \mu \mathrm{m})$, but Figure 1 shows that an additional order-of-magnitude gain is needed to resolve developing planetary systems. Sub-arcsecond resolution is also required to beat extragalactic source confusion and unambiguously distinguish the emissions of individual distant galaxies. (Figure 2c). To achieve the required resolution, a single-aperture telescope would have to be about $40 \mathrm{~m}$ in diameter. A cryogenic telescope this size would be prohibitively expensive and over-designed in terms of lightcollecting capability for the community's science requirements. Quoting Harwit et al., "As amply illustrated by the performance of the small FIR/SMM space telescopes already launched, the light gathering power of telescopes is now somewhat of a secondary concern, given the strong signals and high numbers of photons obtained from astronomical sources and the exquisite sensitivity of detectors developed to date." ${ }^{14}$ An interferometer is an excellent alternative to a large telescope, as it can provide the necessary angular resolution and still have the light collecting power needed to satisfy the sensitivity requirements.

The Space Infrared Interferometric Telescope (SPIRIT) shown in Figure 1 was designed to revolutionize our understanding of the formation of planetary systems and enable us to "follow the water" as these systems develop; to find the telltale signatures of exoplanets in spatially resolved protostellar and debris disks; and to make unique and profound contributions to our understanding of the formation, merger history, and star formation history of galaxies. ${ }^{15,16}$ SPIRIT rotates and its telescopes move along the length of the structure to sample many baselines and provide highquality hyperspectral images. 
Figure 2 illustrates how spatio-spectral interferometry will be used to map spectral line emission in protoplanetary systems, resolve planet-induced structures in dust thermal emission from debris disks, and measure the spectra of individual distant galaxies.

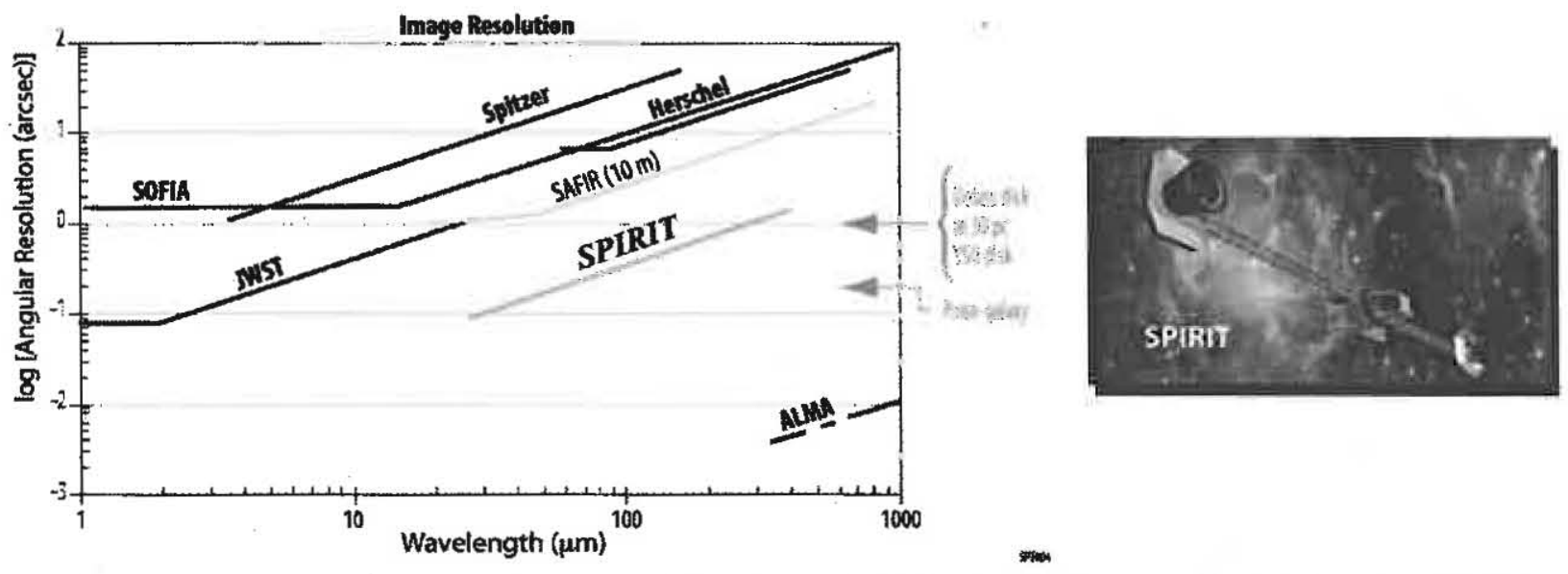

Figure 1. Vast improvements in angular resolution beyond those provided by the current generation of far-IR missions are needed to achieve the astrophysics community's science goals and resolve the sources of interest. The 36-m maximum baseline Space Infrared Interferometric Telescope (SPIRIT; right) matches the resolition of JWST at 10x longer wavelengths $(25-400 \mu \mathrm{m})$.

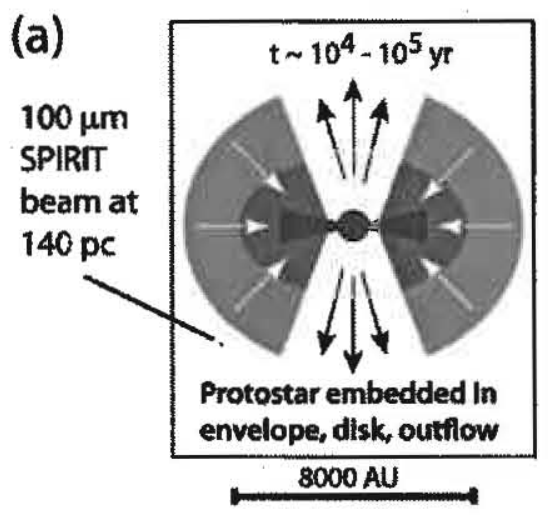

(b)
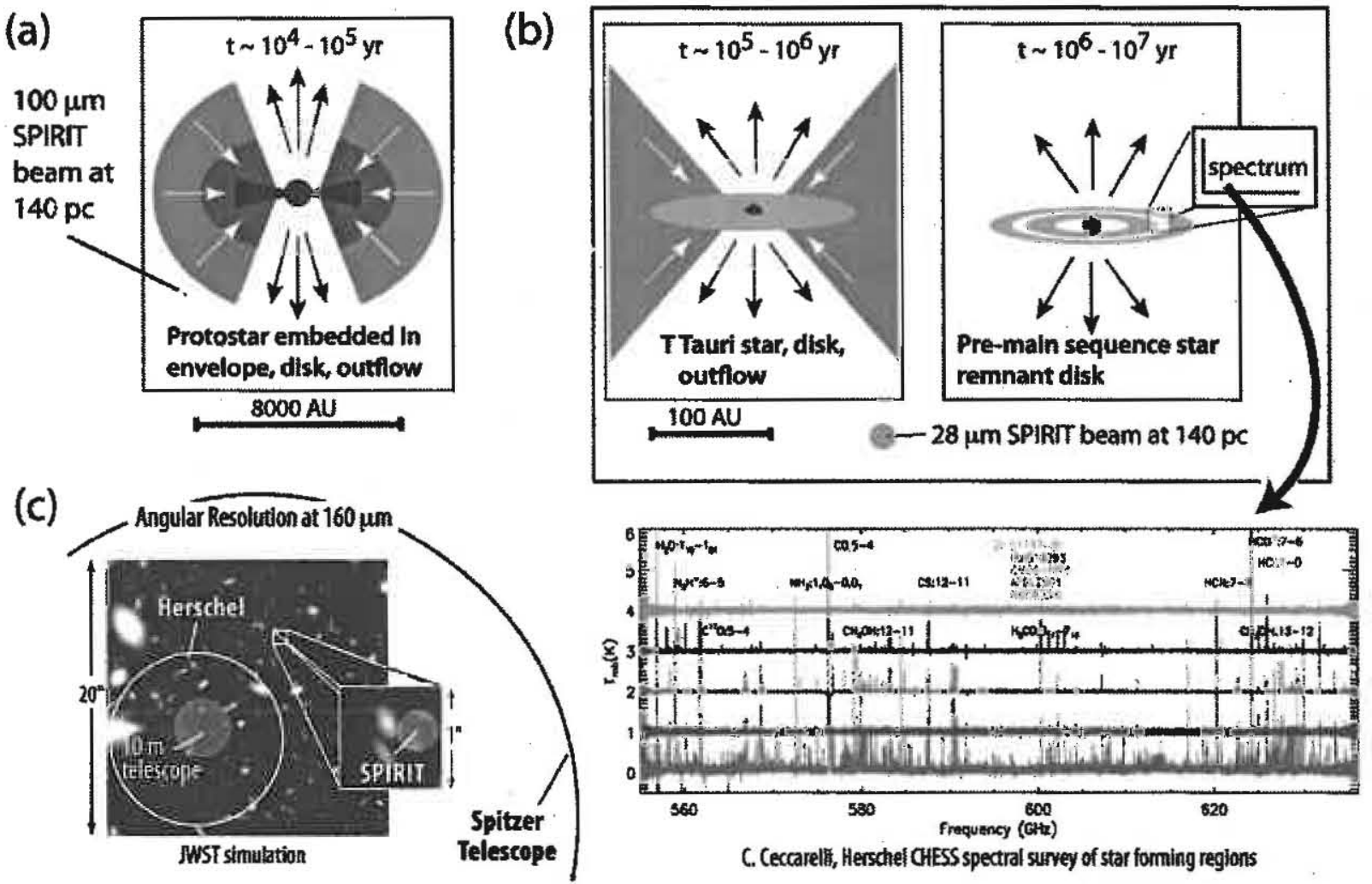

Figure 2. SPIRIT spatially resolves (a) protostars, (b) forming and developed planetary systems, and (c) distant galaxies, while simultaneously measuring their spectra. 
Many observational targets, such as young protostars (Figure 2a), clusters of developing or evolved planetary systems (Figure 2b), or the galaxies in a "deep field" (Figure 2c), fit within a 1 or 2 arcmin FOV and can be observed in parallel with an appropriately designed interferometer. A conventional imaging interferometer has a FOV dictated by the diffraction-limited response of the individual light collecting telescopes, $\theta_{p} \sim \lambda / \mathrm{D}$, for telescope diameter $\mathrm{D}$. For reasonable parameters in the far-IR, $\theta_{p}$ is of the order or arcseconds, considerably smaller than the desired FOV. Radio astronomers call $\theta_{\mathrm{p}}$ the "primary beam" and use "mosaicing" methods to image larger areas. ${ }^{17,18}$ We have been demonstrating and testing the practical limitations of wide field-of-view spatio-spectral optical interferometry in the lab for the past decade. ${ }^{19,20}$

In summary, the "pot of gold at the end of the path" is a deep understanding of the astrophysical and chemodynamic processes inherent in galaxy, star, and planetary system formation, and profound insight into how the conditions for planet habitability arise.

\section{THE FAR-IR COMMUNITY'S VISION OF AN IDEAL PATH, CIRCA 2002}

Motivated by these goals, stirred by the imminent launch of Spitzer, eagerly anticipating Herschel, and presciently peering beyond Herschel, the far-IR astrophysics community convened a series of planning workshops around the turn of the millennium. The community understood that expendable cryogens would limit the operational lifetimes of Spitzer and Herschel and that important astrophysical information would be lacking until the rich measurement capabilities enjoyed by the community at UV/visible wavelengths were attained in the far-IR. Most conspicuously lacking at these long wavelengths was high spatial resolution. With its $3.5 \mathrm{~m}$ diameter primary mirror, Herschel would make a significant stride toward higher resolution, but the essential drivers for sub-arcsecond resolution - those depicted in Figures 1 and 2 - were already known. The community also understood that only a cryogenic observatory in space would offer unimpeded access to the far-IR sky and the sensitivity needed to make the desired measurements. Given the long timescales involved in mission planning and development, there was no time to spare if a next-generation far-IR space observatory would be ready to launch within a few years of Herschel's end of operations.

In the heady days leading up to the 2000 US Decadal Survey in astrophysics, the entire astronomical community set its sights on a variety of ambitious goals. The far-IR community at this time advocated for two missions: a 10-m diameter Single Aperture Far-IR (SAFIR) telescope, and a kilometer maximum baseline spatio-spectral interferometer called the Submillimeter Probe of the Evolution of Cosmic Structure (SPECS). ${ }^{21}$. SAFIR was envisioned as a step toward SPECS. In response, the 2000 Decadal Survey called for "[a] rational coordinated program for space optical and infrared astronomy would build on the experience gained with [JWST] to construct [a JWST-scale filled-aperture far-IR telescope] SAFIR, and then ultimately, in the decade 2010 to 2020 , build on the SAFIR, TPF, and SIM experience to assemble a space-based, far-infrared interferometer."22 SAFIR was recommended for a new start by the end of the 2000 -2010 decade.

A 2002 far-IR community workshop focused on the development of a practical plan that would attain the ultimate goal incrementally. The consensus view of 124 workshop participants was recorded in the Community Plan for FarInfrared/Submillimeter Space Astronomy. ${ }^{23}$ In addition to the flagship missions SAFIR and SPECS, the Community Plan recommended investment in eight specific mission-enabling technologies, relatively small missions, and the structurallyconnected SPIRIT interferometry mission as a scientific precursor to the formation flying SPECS, if SPIRIT's cost were determined to be much less than that of a flagship mission. Like all large missions, clearly SAFIR and SPECS are multibillion US\$ observatories.

Figure 3 depicts the far-IR space mission roadmap envisioned in the Community Plan. The plan proved to be robust, as it was revisited in additional community workshops and discussions leading up to the 2010 Decadal Survey, after SPIRIT, SAFIR and SPECS mission concept studies were conducted, and deemed still to be the best path forward. ${ }^{2}$ 
The US Far-IR Community Plan, c. 2002

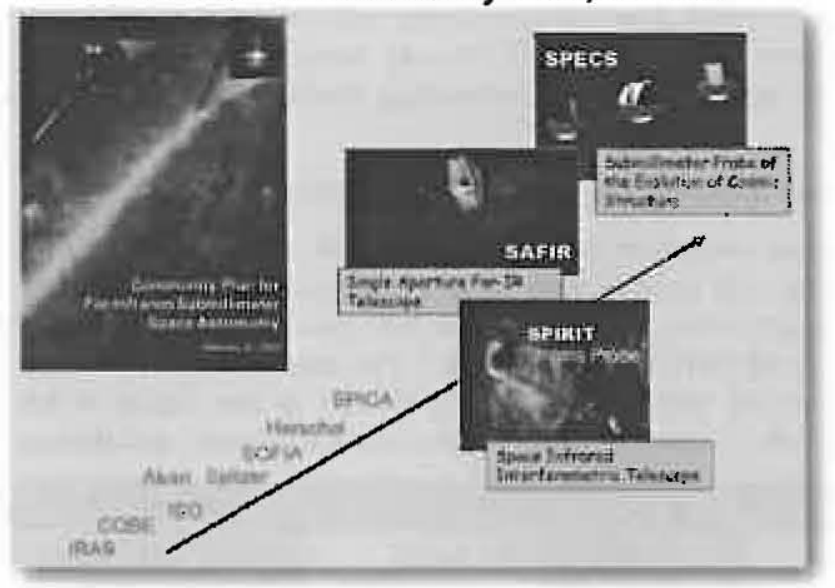

Figure 3. Since 2002 the far-IR community in the US has been steadfast in calling for a progression of space missions, ultimately culminating in the deployment of a kilometer maximum-baseline formation flying interferometer. The structurally connected interferometer SPIRIT is recognized as a logical scientific and technical stepping stone on the path.

\section{TRAVERSING THE REAL WORLD}

When the far-IR community's plan is overlaid on the landscape of the real world, the optimal path warps and stretches, but it remains viable and compelling. This section gives an overview of tantalizing science highlights, far-IR mission concept studies, and technological progress from the past decade, and it introduces new route markers (the Japanese mission SPICA and balloon-borne interferometers) and characterizes the current landscape.

\subsection{Spitzer and Herschel illuminate the road ahead}

Spitzer operated in the far-IR until its cryogen ran out in May 2009, fortuitously a mere two months before Herschel made its "first light" observations. Both missions have been enormously productive, as suggested in Section 2.1. They also foretell the scientific bounty that will lie untapped until we capture higher resolution views of the far-IR sky with interferometers. To make the point, we offer two examples. First, the blended light of myriad galaxies seen in the Herschel Multi-tiered Extragalactic Survey (HerMES) ${ }^{24}$ tempts one to ask what lies beneath the confusion noise floor. How did galactic star formation and nuclear activity, mergers and interactions evolve over time? When did the first dust appear, permanently altering, likely catalyzing, and optically concealing star formation? Second, Spitzer and Herschel were able to image a handful of debris disks, the dusty remnants of planet formation. Dust concentrations arise in these disks when dust grains interact gravitationally with planets and become "trapped" in resonant structures. ${ }^{9}$ Future far-IR interferometers will image many debris disks, find many still-undiscovered planets, and help to characterize extrasolar planetary systems.

\subsection{Clearer visions of the future: mission concept studies}

Several far-IR interferometry mission concepts were studied during the past decade, providing important opportunities for the development of Design Reference Missions (DRM1) and derivation of the flowdown from science requirements to measurement requirements to engineering requirements. Technology needs were brought into sharp focus. Tradeoff analyses were conducted and alternative implementation approaches were explored. In the US, NASA studied SPIRIT as a candidate Origins Probe ${ }^{15,16}$ and SPECS as a "vision mission."14 In Europe, two concepts were proposed for the FarInfrared Interferometer (FIRI): the heterodyne system ESPRIT ${ }^{25}$ and a spatio-spectral optical interferometer along the lines of SPECS ${ }^{26}$ ESTEC conducted a Technology Reference Study for FIRI, choosing a single-spacecraft, structurally connected design concept for study. ${ }^{27}$

In 2004-05, SPIRIT was studied extensively by an integrated science and engineering team. A detailed DRM was prepared, and the mission concept matured through three design cycles, each of which was assessed for performance relative to the DRM. Grass roots and independent parametric cost estimates were developed for each mission design. Compromises were made in measurement capability in the interest of hitting a Probe-class cost target, and engineering 
studies grew progressively sophisticated, culminating after a year of intensive, coordinated effort in detailed design reports, development plans, Integration \& Test (I\&T) concepts, and a technology roadmap. . $^{15,28,29,30,31,32}$ By the end of the decade, SPIRIT was ready to recommend to the 2010 Decadal Survey, and a launch date in 2023 was targeted ${ }^{\text {i6 }}$ The total lifecycle cost of the SPIRIT mission, including technology development, was estimated to be $\$ 1.25 \mathrm{~B}$ in FY09 US dollars.

\subsection{A glance in the rearview mirror: a decade of technological progress}

The key enabling technologies for wide-field spatio-spectral far-IR interferometry are well understood and comprise principally: detectors; cryocoolers and cryo-thermal system integration and test; and the wide-field "double Fourier" (spatio-spectral) technique. ${ }^{33}$ Figure 4 shows the 2009 status and plan for technology development as it appeared in the SPIRIT white paper submitted to the 2010 Decadal Survey. ${ }^{16}$ The requested augmentation in technology funding hasn't materialized, so recent progress has been slower than indicated in the Figure 4 timeline. However, respectable technological progress was made for a variety of purposes over the past decade, and the key technologies are maturing.

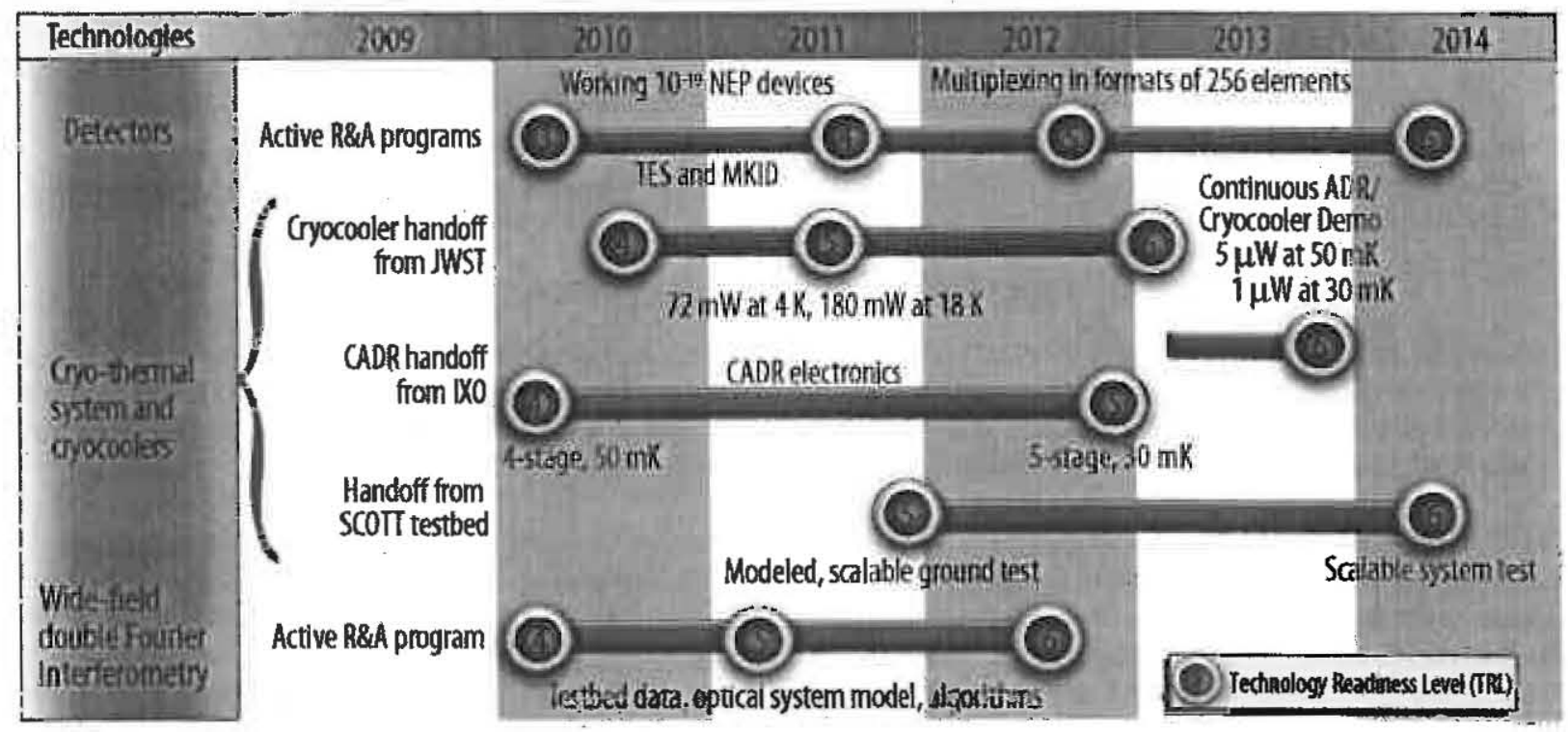

Figure 4. The technology development plan for SPIRIT would build on existing heritage and past investments to mature all the key mission-enabling technologies to Technology Readiness Level (TRL) 6 in four years.

\subsubsection{Detector technology}

The detector technology needed for SPIRIT was described in a technology paper submitted to the Decadal Survey by Bock et al. ${ }^{34}$ SPIRIT requires detector arrays with modest pixel count, the largest array being $14 \times 14$ pixels. The detector sensitivity (Noise Equivalent Power, NEP) must be about $10^{-19} \mathrm{~W} / \mathrm{Hz}^{1 / 2}$. The detectors must also have a time constant $\tau \sim 185 \mu$ s or faster, and a dynamic range dynamic range (ratio of sky brightness to noise) of at least 2500 .

Leg-isolated superconducting transition edge sensor (TES) bolometers are presently the most mature technology that can meet the SPIRIT requirements. TES bolometers saw first light at the IRAM telescope in $2007,{ }^{35}$ and the GISMO instrument in which they are deployed is now scientifically productive. ${ }^{36}$ Although these detectors currently fall short of the SPIRIT response time requirement by a factor of $\sim 2000,{ }^{37}$ scaling from present performance suggests that the existing technology can meet the sensitivity, array size, and possibly also the time constant requirements if the detectors are operated at $30 \mathrm{mK} \cdot{ }^{38,39}$ Recent measurements of TES bolometers that use electron-phonon decoupling demonstrate that these detectors can meet all of SPIRIT's requirements. ${ }^{40,41}$ Kinetic Inductance Detectors also have demonstrated potential for high performance, and multiple research groups have made significant progress with these devices in recent years. ${ }^{42,43,44}$ 


\subsubsection{Cryo-thermal system and cryo-cooler technology}

The technological challenge in this area consists of: (1) upgrading the JWST cryocooler to enable higher power cooling to $4 \mathrm{~K}$; (2) upgrading the International X-ray Observatory (IXO) Continuous Adiabatic Demagnetization Refrigerator (ADR) to enable detector cooling to $30 \mathrm{mK}$; and (3) integrating the coolers into a functional cryo-thermal system and demonstrating system performance.

Cryo technology development during the past decade was driven by JWST and IXO. This represents a leveraging opportunity for far-IR interferometry. The JWST MIRI cryocooler being readied for flight in 2018 is required to provide $60 \mathrm{~mW}$ of cooling at $6 \mathrm{~K}$ and $75 \mathrm{~mW}$ of cooling at $18 \mathrm{~K}$, and the Continuous Adiabatic Demagnetization Refrigerator (CADR) under development for the IXO is adaptable to meet the SPIRIT focal plane cooling requirements. ${ }^{31}$

Thermal system verification for SPIRIT will be challenging because the thermal balance test will contain warm, $\sim 300 \mathrm{~K}$, and cold, $4 \mathrm{~K}$, components, and test chamber non-idealities can dominate the results. For example, the Spitzer thermal balance test showed a measured heat leak of $52 \mathrm{~mW}$ into the instrument when the on-orbit value turned out to be only 6 $\mathrm{mW}$. To improve this situation, we conducted an experiment using an $18 \%$ scale model of a preliminary SPIRIT telescope design, the Subscale Cryo-thermal Testbed (SCOTT). ${ }^{45}$ The test results agreed remarkably well with pre-test thermal model predictions, proving out the design and giving us confidence that SPIRIT's telescopes and instrument module can be cooled with JWST-like cryocoolers.

\subsubsection{Wide-field spiatio-spectral interferometry}

As detailed in papers prepared for the parallel SPIE conference on Optical and Infrared Interferometry, significant progress was made during the past decade in developing and testing the practical limitations inherent in the wide-field spatio-spectral interferometric technique ${ }^{20}$ and the algorithm and software needed to process spatio-spectral interferometric data and derive hyperspectral data cubes. ${ }^{46}$ This "technology" has matured to TRL 5. Experiments were conducted with a laboratory testbed, the Wide-field Imaging Interferometry Testbed (WIIT). WIIT in its current operating environment is functionally equivalent to SPIRIT operating in space. WIIT also emulates SPIRIT by producing representative interferometric data. A high-fidelity analytical model of the testbed was shown to yield synthetic data that closely match data obtained in the lab, demonstrating a good understanding of testbed performance and instrumental fringe visibility loss and noise terms. Recent spatial-spectral synthesis results are shown in Figure 5.

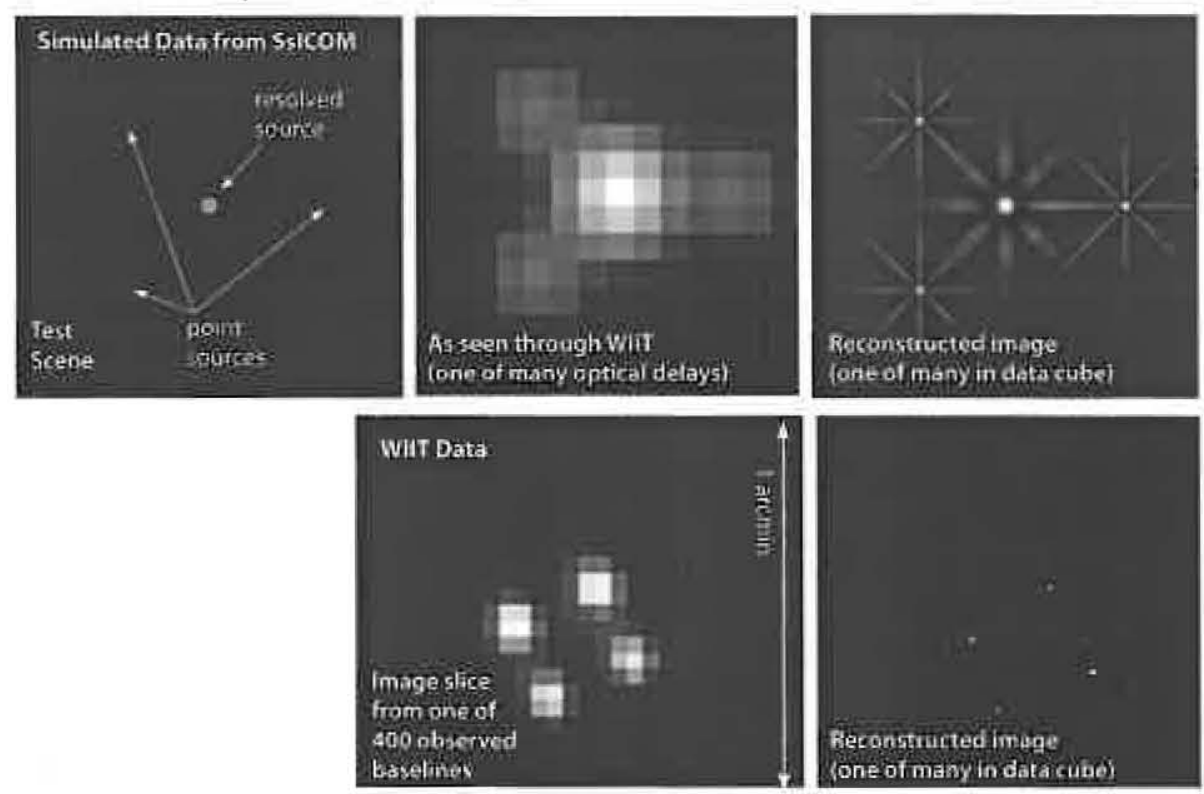

Figure 5. Spatio-Spectral Synthesis Software (S4) processing of synthetic interferometric data from an analytical model (top row) and WIIT (bottom row). Only $0,45,90$, and $135 \mathrm{deg}$ baseline angles were used in the top, accounting for the starshaped "dirty beam." CLEAN deconvolution can be used to remove these artifacts, but they only appear when the u-v plane is sparsely sampled. Each reconstructed image (right) is one of many drawn from the $\$ 4$ output hyperspectral cube representing the test scene (top) or the scene that WIIT observed with many baselines (bottom). 


\subsubsection{Additional relevant technological developments}

European investments in-metal mesh filter technology for Herschel are directly applicable to future far-IR interferometry missions. $^{47}$

Mechanisms similar to those used on SPIRIT have flown successfully on past NASA and ESA missions. For example, cryogenic delay lines were flown in COBE/FIRAS and Cassini/CIRS. More recently, TNO in The Netherlands developed a precision delay line for Darwin. ${ }^{48}$

Although metrology system components developed for the Space Interferometry Mission (SIM) ${ }^{49}$ vastly exceed SPIRIT requirements, one might expect that a small additional investment could be made to realize cost savings associated with greatly relaxed tolerances.

The SPIRIT telescope transport mechanism could be similar to the trolley-on-rails device used in the Mobile Transporter on the International Space Station. Again, cost savings can be realized because ISS-related human safety factors will not pertain to SPIRIT.

\subsubsection{Technology for SPECS}

In addition to the SPIRIT technologies discussed above, tethered formation flight $^{50}$ and faster, more sensitive detectors will be needed for SPECS. ${ }^{14} \mathrm{~A}$ tethered formation flight testbed was developed for the ISS and tested on the ground. ${ }^{51}$ Untethered formation flight has been tested on the ISS, but the tether adapters have not yet flown.

\subsection{The rocky road ahead}

\subsubsection{A fellow traveler: SPICA}

When the US far-IR community formulated its vision in 2002, the Japanese Space Infrared Telescope for Cosmology and Astrophysics (SPICA) ${ }^{52}$ was recognized as important scientifically and as a driver for detector technology. ${ }^{23}$ In 2008 , under the assumption that SPICA would be launched in 2017, the community chose instrument involvement in SPICA as its most urgent priority. ${ }^{2}$ The 2010 Decadal Survey adopted the SPICA recommendation, capped US involvement at $\$ 150 \mathrm{M}$, and stipulated that the endorsement was contingent on there being room in NASA's budget after higher priorities were satisfied. ${ }^{1}$

ESA's potential contribution to SPICA includes the telescope and thus could be critical to Japan's acceptance of the mission proposal. A European decision on participation is expected soon. The current proposal calls for launch in 2022 , five years later than assumed in US community planning.

\subsubsection{The era of tight budgets and shrinking ambitions}

The 2010 Decadal Survey Committee was asked by NASA to factor realistic mission costs into its deliberations and to deliver an executable plan for space astrophysics. Going in, the community was restrained, as NASA's budget outlook was such that no new flagship mission could be started until late in the decade. Accordingly, less ambitious space missions were proposed than those suggested a decade earlier. In addition to asking for US involvement in SPICA, the far-IR Community Plan made this request: "(i) to prepare the technical prerequisites in the $2010-2020$ decade and (ii) to conduct phase-A studies of both SAFIR and SPIRIT before the end of the decade, to provide the 2020 Decadal Review with a strong recommendation on which of these two missions has greater immediate astronomical promise and higher readiness for early launch in the 2020 - 2035 era." At an earlier time, such investments in technology and mission concept studies would have been considered affordable and prudent. New mission studies would refresh the Design Reference Missions to take into account a decade of scientific progress based on observations made with Spitzer, Herschel, and other telescopes. The New Worlds, New Horizons report made a modest set of recommendations relative to past decadal surveys, the tentative commitment to SPICA mentioned above being the only one that aligned with the far-IR Community Plan. ${ }^{1}$

\subsubsection{The impending hiatus: a community at the precipice}

Only a cold telescope in space can provide sensitive far-IR measurements rivaling those available today. Between 2013, when Herschel's cryogen supply runs out, and 2022, the earliest conceivable year for a SPICA launch, no far-IR data will stream to the astrophysics community from space. SPICA will be comparable to Herschel in size and much colder and more sensitive, but it will not satisfy the requirement for sub-arcsecond angular resolution discussed in Section 2. 
Neglect will come at a price. Starting this year, the community will enter free fall. The founding fathers of space far-IR astrophysics are approaching retirement, retired, or alas in some cases live only in our fond memories. Far-IR instrumentalists and astrophysicists will be forced to seek employment in more vibrant fields, and fewer young people will enter the field. In the coming decade, three decade's worth of accumulated technical acumen will evaporate. Eventually, a new generation of technical experts will require training, and costly mistakes may be made, as old lessons are re-learned. Tragically, this gloomy scenario is today's reality.

Will suborbital platforms sustain the technical expertise needed for space-based far-IR interferometry? No. The Stratospheric Observatory for Infrared Astronomy (SOFIA) is an instrument testbed and a fine platform for certain measurements, such as high-resolution spectroscopy. Unfortunately, SOFIA doesn't drive the technology needed for a space mission, such as detectors with extraordinary sensitivity. Balloon and laboratory experiments are significant training opportunities, but the budgets for such projects are two orders of magnitude smaller than those of space missions, and a proportionately small number of people gain experience.

\section{THE PATH AHEAD}

Despite the hurdles that now lie in the path to far-IR interferometry in space, long-term prospects for an interferometry mission are excellent: the science case is compelling and durable, the relevant technologies are approaching TRL 6, and eventually a mission like SPIRIT will be affordable.

\subsection{Completing the technology development}

The first priority of those who share our vision is to complete development of the mission-enabling technologies shown in Figure 4. We must demonstrate to the broader scientific community that the technology is in hand and that wide-field spatio-spectral optical interferometry can affordably satisfy the community's high-resolution measurement requirements in the far-IR.

Prudence calls for continued parallel investment in competing detector technologies, leading to down-selection to the one detector type that exhibits the greatest promise at an appropriate time. This approach worked for JWST and will assure the timely delivery of detectors for SPICA and SPIRIT.

Cooling to $\sim 4 \mathrm{~K}$ can be achieved by substituting ${ }^{3} \mathrm{He}$ for ${ }^{4} \mathrm{He}$ as the working fluid in the lowest temperature stage of the JWST cryocooler. This demonstration will advance the cryocooler TRL to 5 for SPIRIT. To achieve TRL 6 for SPIRIT, the CADR, when interfaced with a mechanical cryocooler operating at 4-6 K, must demonstrate the capacity to lift $5 \mu \mathrm{W}$ of heat at $50 \mathrm{mK}$ or $\sim 1 \mu \mathrm{W}$ at $30 \mathrm{mK}$. Already, the CADR has demonstrated continuous cooling of $6 \mu \mathrm{W}$ at $50 \mathrm{mK}$ and $1.5 \mu \mathrm{W}$ at $35 \mathrm{mK}$ starting from a heat sink temperature of $5 \mathrm{~K}$, nearly meeting the SPIRIT requirement. To progress to TRL 5 the CADR development program will take about three years, resulting in a brassboard, demonstrating an integrated CADR/cryocooler system capable of satisfying the SPIRIT focal plane cooling requirements. The CADR for SPIRIT will have 4 stages operating between roughly $5 \mathrm{~K}$ to $1.2 \mathrm{~K}, 1.2 \mathrm{~K}$ to $0.3 \mathrm{~K}, 0.3 \mathrm{~K}$ to $0.05 \mathrm{~K}$ and a stage that operates continuously at $0.05 \mathrm{~K}$. A fifth stage will operate continuously at about $1.2 \mathrm{~K}$ to provide a low thermal emission environment surrounding the detectors to eliminate stray thermal radiation and to provide a stable cooling stage for detector amplifiers. The nominal input power varies between 30 and $80 \mathrm{~W}$, depending on the cooling power required. The CADR will reject approximately $3 \mathrm{~mW}$ to $4 \mathrm{~K}$, a very modest cooling power for the current suite of TRL 4-5 cryocoolers. New thermal system verification tests similar to those conducted with the SCOTT testbed ${ }^{37}$ will have to be conducted with the integrated CADR/cryocooler system.

At the current pace, two more years of effort will be sufficient to mature wide-field spatio-spectral interferometry for SPIRIT to TRL 6. A hyperspectral scene generator recently installed in the WIIT testbed will enable inteferometric observations of astronomically representative test scenes. ${ }^{53}$

\subsection{Pathfinding: balloon interferometry}

When the path to the summit of a mountain is too steep to climb directly, one sometimes takes a route with switchbacks. For the programmatic reasons outlined in Section 4.5, the path to space-based far-IR interferometry will be forced to meander.

The Balloon Experimental Twin Telescope for Infrared Interferometry (BETTII), now in development, is an 8-meter baseline far-IR interferometer designed to fly on a high altitude balloon. ${ }^{54}$ BETTII uses a double-Fourier Michelson interferometer to obtain spatial and spectral information on science targets. The beam-combining instrument in BETTII 
is quite similar to the instrument in SPIRIT. BETTII will test fringe-tracking on near-IR sources and provide arcsecond to sub-arcsecond views of selected, bright far-IR sources.

Balloon-based interferometry would lie on the path of steepest ascent to far-IR interferometry in space were it not for the fact that the greatest technical challenges in each case barely overlap. Unlike SPIRIT, which will operate in a quiescent environment, BETTII will swing like a pendulum and its support structure will sag in l-g. To compensate continually varying external optical path length differences, BETTII requires fast fringe and angle tracking and a long-stroke optical delay line. SPIRIT will be oriented with its baseline vector perpendicular to the line of sight, keeping external optical path length differences small and path length variations slow. SPIRIT must be compact for launch and deployable, whereas BETTII needn't use space efficiently. SPIRIT's telescopes and instrument must be cryocooled; BETTII will operate at ambient temperature and never reach thermal equilibrium. SPIRIT, requires sensitive detectors to take advantage of the low photon background in space. BETTII's sensitivity is limited by the warm atmosphere and optics; its detectors needn't be especially sensitive. BETTII will be serviceable between flights, whereas SPIRIT will be inaccessible in a Sun-Earth L2 orbit. The Japanese balloon-borne Far-Infrared Telescope Experiment (FITE) ${ }^{55}$ is scheduled to fly before BETTII, but FITE uses Fizeau (image plane) beam combination, differing from SPIRIT more fundamentally.

BETTII was a proposed as a hedge in case NASA's investment in SPIRIT would be delayed. That proved to be a wise decision. Despite the divergent technical challenges between balloon-borne and spaced-based inteferometers, BETTII and FITE may whet the community's appetite for a space-based interferometer.
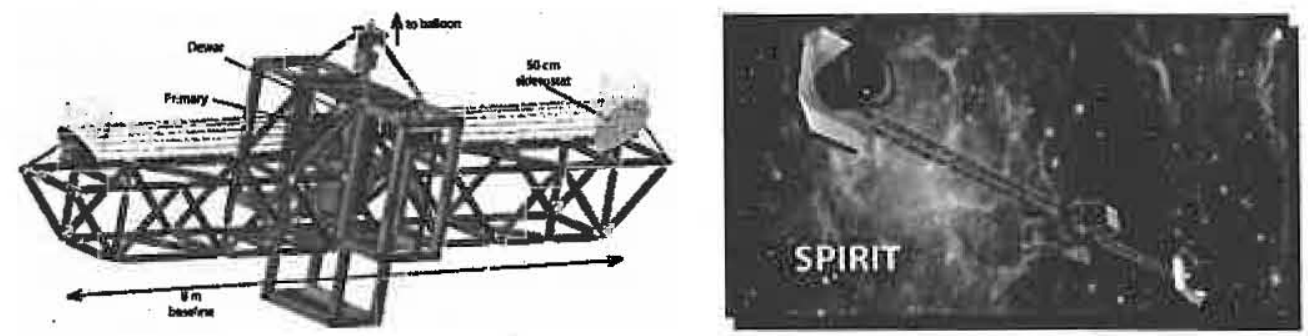

Figure 6. (left) BETTII has a fixed $8 \mathrm{~m}$ interferometric baseline, samples only a few spatial Fourier components in the $30-90$ $\mu \mathrm{m}$ spectral range, and provides spectral resolution $\Delta \lambda / \lambda \sim 20$. (right) SPIRIT densely samples the $u-v$ plane and has a $36 \mathrm{~m}$ maximum baseline to provide high-quality images and $\Delta \lambda / \lambda \sim 3000$ spectroscopy at wavelengths from 25 to $400 \mu \mathrm{m}$.

\subsection{Lifting the fog: US and European decisions on participation in SPICA}

The path to far-IR interferometry in space will become clearer when decisions regarding participation in SPICA are reached in the US and Europe. SPICA will either fly as planned, driving technology in a direction helpful to interferometry, or fly later or not at all, widening the already gaping hole between Herschel and its successor in far-IR space astrophysics.

\subsection{All aboard! - a positive outlook for international partnership}

Since 1998, a succession of far-IR community planning workshops in the US, Canada, and Europe has led to the emergence of new ideas and a consensus in favor of interferometry. This global harmony arose from parallel methodical assessments of scientific objectives and measurement requirements. Sub-arcsecond resolution is needed, but a singleaperture telescope tens of meters in diameter simply isn't necessary to accomplish the community's science goals, even if such a thing were technically feasible and affordable. Interferometry is feasible and affordable. Research groups in the US, Europe, Japan, and Canada have been informally coordinating and collaborating for over a decade. For example, the UK has assembled a double-Fourier testbed that operates at far-IR wavelengths, in part to test quasi-optical metal mesh components (dichroic filters and beamsplitters) suitable for use in the SPIRIT beam combiner. ${ }^{56}$ The Cardiff University group plans to contribute filters and beamsplitters to the Goddard group for use in BETTII. The wide-field imaging interferometry group at Goddard is hosting a graduate student from Cardiff, publicly releases data from the WIIT testbed, and often consults with groups in Europe and Canada. The BETTII and FITE teams in the US and Japan exchange technical information and learn from each other. It seems plausible to us that the far-IR community will be ready to unite in a consortium and respond with an interferometry mission proposal when the appropriate funding opportunity appears. 


\subsection{The light turns green: Probe and M-class mission opportunities}

There is growing interest in the US in the creation of a Probe-class mission line for astrophysics, where a "Probe" could be in the $\$ 650 \mathrm{M}$ to $\$ 1 \mathrm{~B}$ range. In Europe, ESA's M class missions are capped at 470 MEuro, or about $\$ 590 \mathrm{M}$ in US dollars. A NASA-ESA Probe/M partnership could pay for a mission like SPIRIT.

\section{CONCLUSION}

A question that captures the public's imagination, inspires children, and motivates astrophysicists will remain out of reach until we launch a far-IR spatio-spectral interferometer: How did we get here? Sensitive, high-resolution spatial and spectral information in the far-IR is essential to understand merger-driven star formation and chemical enrichment in galaxies, star and planetary system formation, and the development and prevalence of water-bearing planets.

Through determined efforts around the world, the key enabling technologies for space-based far-IR interferometry are approaching maturity, and pathfinding balloon projects are underway.

Although a budget-driven programmatic fog has descended on the path to far-IR interferometry in space, the fog eventually will lift. When that occurs, space-based far-IR interferometry will be the right idea at the right time. In the mean time, in our opinion, it is simply "the right idea."

\section{REFERENCES}

[1] New Worlds, New Horizons in Astronomy and Astrophysics, Committee for a Decadal Survey of Astronomy and Astrophysics, National Research Council, The National Academies Press, $<$ http://www.nap.edu/openbook.php?record_id=12951> (2010). http://www.nap.edu/openbook.php?record id=12951

[2] Harwit, M. et al., "Far-Infrared/Submillimeter Astronomy from Space: Tracking an Evolving Universe and the Emergence of Life," the Far-IR Community Plan white paper submitted to the Astronomy and Astrophysics Decadal Survey of 2010, <http://www.ipac.caltech.edu/pdf/FIR-SMM_Crosscutting_Whitepaper.pdf $>$ (2008). http://www.ipac.caltech.edu/pdf/FIR-SMM Crosscutting_Whitepaper.pdf

[3] Nisini, B. et al., "Far infrared mapping of the gas cooling along the L1448 outflow," A\&A, 360, 297 (2000).

[4] van Dishoeck, E.F., "ISO Spectroscopy of Gas and Dust: From Molecular Clouds to Protoplanetary Disks," ARA\&A, 42, 119 (2004).

[5] Ceccarelli, C. et al., "Herschel spectral surveys of star-forming regions: Overview of the 555-636 GHz range ," A\&A 521, L22 (2010).

[6] Sturm, B. et al., "First results of the Herschel key program "Dust, Ice and Gas In Time" (DIGIT): Dust and gas spectroscopy of HD100546," A\&A, 518, L129 (2010).

[7] Hogerheijde, M.R. et al., "Detection of the Water Reservoir in a Forming Planetary System," Science, 334, 338 (2011).

[8] Ford, K., Saavik, E., and Neufeld, D.A., "Water Vapor in Carbon-rich Asymptotic Giant Branch Stars from the Vaporization of Icy Orbiting Bodies," ApJ, 557, L113 (2001).

[9] Moran, S.M., Kuchner, M.J., and Holman, M.J., "The Dynamical Influence of a Planet at Semijamor Axis 3.4 AU on the Dust around $\varepsilon$ Eridani," ApJ, 612, 1163 (2004).

[10]Lutz, D., Veilleux, S., and Genzel, R., "Mid-Infrared and Optical Spectroscopy of Ultraluminous Infrared Galaxies: A Comparison," ApJ, 517, 13L (1999).

[11]Thornley, M.D. et al., "Massive Star Formation and Evolution in Starburst Galaxies: Mid-infrared Spectroscopy with the ISO Short Wavelength Spectrometer," ApJ, 539, 641 (2000).

[12] Fischer, J. et al., "Herschel-PACS spectroscopic diagnostics of local ULIRGs: Conditions and kinematics in Markarian 231," A\&A, 518, L41 (2010).

[13] Stacey, G.J. et al.., "The 158 micron forbidden C II line - A measure of global star formation activity in galaxies," ApJ, 373, 423 (1991).

[14]Harwit, M. et al., "A Kilometer-Baseline Far-Infrared/Submillimeter Interferometer in Space," in Progress in Astronautics \& Aeronautics, vol. 224, M.S. Allen, ed. (AIAA: Reston, VA), pp. 301 - 326 (2008).

[15]Leisawitz, D. et al., "The Space Infrared Interferometric Telescope (SPIRIT): High-resolution imaging and spectroscopy in the far-infrared," Adv. Sp. Res., 40, 689 (2007) 
[16]Leisawitz, D. et al., "The Space Infrared Interferometric Telescope (SPIRIT): A Far-IR Observatory for Highresolution Imaging and Spectroscopy, white paper submitted to the Astronomy and Astrophysics Decadal Survey of 2010, <http://astrophysics.gsfc.nasa.gov/cosmology/spirit/ > (2009). http://astrophysics.gsfc.nasa.gov/cosmology/spirit/

[17] Cornwell, T. J., "Radio-interferometric imaging of very large objects," A\&A, 202, 316 (1988).

[18] Bhatnagar, S., Golap, K., and Cornwell, T. J., "Mosaicing with interferometers: An Efficient Algorithm for Imaging and Image Plane Corrections," in Astronomical Data Analysis Software and Systems XIV, Shopbell, P., Britton, M. \& Ebert R., eds., ASP Conference Series, 347, 96 (2005).

[19]Leisawitz, D.T. et al., "Wide-field imaging interferometry testbed I: purpose, testbed design, data, and synthesis algorithms," in Interferometry in Space, M. Shao, ed., Proc SPIE, 4852, 255 (2003).

[20]Leisawitz, D. et al., "Developing wide-field spatio-spectral interferometry for far-infrared space applications," in Optical and Infrared Interferometry III, F. Delplancke, J.K. Rajagopal \& F. Malbet, eds., Proc. SPIE 8445 (2012).

[21] Mather, J.C. et al., "The Submillimeter Frontier: A Space Science Imperative," <http://arxiv.org/abs/astro$\mathrm{ph} / 9812454>$ (1998). http://arxiv.org/abs/astro-ph/9812454

[22] Astronomy and Astrophysics in the New Millennium, Astronomy and Astrophysics Survey Committee, National Research Council, The National Academies Press, <http://www.nap.edu/openbook.php?isbn=0309070317> (2001). http://www.nap.edu/openbook.php?isbn=0309070317

[23] "Community Plan for Far-IR/Submillimeter Space Astronomy," in New Concepts for Far-Infrared and Submillimeter Space Astronomy, Proceedings of the Second Workshop on New Concepts for Far-IR/Submillimeter Space Astronomy, held March 7-8, 2002 at the University of Maryland Inn and Conference Center, College Park, MD, D.J. Benford \& D.T. Leisawitz, eds., NASA CP-2003-212233, Rept-2003-03082-0, $<$ http://safir.gsfc.nasa.gov/docs/Community_Plan_printed.pdf> (2003). http://safir.gsfc.nasa.gov/docs/Community Plan printed.pdf

[24] Oliver, S.J. et al., "The Herschel Multi-tiered Extragalactic Survey: HerMES," MNRAS, in press $<$ http://arxiv.org/abs/1203.2562> (2012). http://arxiv.org/abs/1203.2562

[25] Wild, W. et al., "ESPRIT: a study concept for a far-infrared interferometer in space," in Optical and Infrared Interferometry, M. Schöller, W.C. Danchi \& F. Delplancke, eds., Proc. SPIE, 7013, 70132R (2008).

[26] Helmich, F. and Ivison, R., "FIRI - a Far-InfraRed Interferometer for ESA," Experimental Astronomy, 23, 245 (2009).

[27]Lindberg, R. et al., "The challenges posed by future far-IR and sub-mm space missions: an overview," in Space Telescopes and Instrumentation 2008: Optical, Infrared, and Millimeter, J.M. Oschmann, Jr., M.W.M. de Graauw \& H.A. MacEwen, eds., Proc. SPIE 7010, 701023 (2008).

[28]Hyde, T.T., Leisawitz, D.T., DiPietro, D., and Rinehart, S.A., "System engineering the Space Infrared Interferometric Telescope (SPIRIT)," in UV/Optical/IR Space Telescopes: Innovative Technologies and Concepts III, H.A. MacEwen \& J.B. Breckinridge, eds., Proc. SPIE, 6687, 66870A-66870A-12 (2007).

[29] Wilson, M.E. et al., "The Space Infrared Interferometric Telescope (SPIRIT): Optical system design considerations," in UV/Optical/IR Space Telescopes: Innovative Technologies and Concepts III, H.A. MacEwen \& J.B. Breckinridge, eds., Proc. SPIE, 6687, 66870B-66870B-11 (2007).

[30]Budinoff, J.G. et al., "Mechanical design of the Space Infrared Interferometric Telescope (SPIRIT)," in UV/Optical/IR Space Telescopes: Innovative Technologies and Concepts III, H.A. MacEwen, \& J.B. Breckinridge, eds., Proc. SPIE, 6687, 66870C-66870C-11 (2007).

[31]DiPirro, M.J., Cottingham, C., Boyle, R., Ollendorf, S., and Leisawitz, D.T., "The SPIRIT thermal system," in UV/Optical/IR Space Telescopes: Innovative Technologies and Concepts III, H.A. MacEwen \& J.B. Breckintidge, eds., Proc. SPIE, 6687, 66870D1 - 11 (2007).

[32] Benford, D.J., Rinehart, S.A., Hyde, T.T., and Leisawitz, D.T., "Cryogenic far-infrared detectors for the Space Infrared Interferometric Telescope (SPIRIT)," in UV/Optical/IR Space Telescopes: Innovative Technologies and Concepts III, H.A. MacEwen \& J.B. Breckinridge, eds., Proc. SPIE, 6687, 66870E-66870E-12 (2007).

[33] Mariotti, J.-M., and Ridgway, S.T., "Double Fourier spatio-spectral interferometry - Combining high spectral and high spatial resolution in the near infrared," A\&A, 195, 350 (1988).

[34]Bock, J. et al., "Bock, J.J. et al. "Superconducting Detector Arrays for Far-Infrared to mm-Wave Astrophysics," an Astro2010 Technology White Paper, <http://www.ipac.caltech.edu/pdf/Bock_scarrays_TEC_OIR_RMS_PSC.pdf> (2009). http://www.ipac.caltech.edu/pdf/Bock scarrays TEC OIR RMS PSC.pdf

[35] Staguhn, J. et al., "GISMO, a 2 mm Bolometer Camera Optimized for the Study of High Redshift Galaxies," J. Low Temp. Phys., 151, 709 (2008). 
[36] Staguhn, J.G. et al., "Latest Results from GISMO: a 2-mm Bolometer Camera for the IRAM 30-m Telescope," EAS Pub. Ser., 52, 267 (2011).

[37] Kenyon, M. et al., "Electrical Properties of Background-Limited Membrane-Isolation Transition-Edge Sensing Bolometers for Far-IR/Submillimeter Direct-Detection Spectroscopy," J. Low Temp. Phys., 151, 112 (2008).

[38] Benford, D.J., Rinehart, S.A., Hyde, T.T., and Leisawitz, D.T., "Cryogenic far-infrared detectors for the Space Infrared Interferometric Telescope (SPIRIT)," in UV:Optical'IR Space Telescopes: Innovative Technologies and Concepts III, H.A. MacEwen \& J.B. Breckinridge, eds., Proc. SPIE, 6687, 66870E1 \&151; 12 (2007).

[39] Day, P. et al., "Distributed Antenna-coupled TES for FIR detector arrays," J. Low Temp. Phys., 151,477 (2008).

[40] Barrentine, E. M. et al., "Sensitivity Measurements of a Transition- Edge Hot-Electron Microbolometer for Millimeter-Wave Astrophysical Observations," J. Low Temp. Phys., 151, 173 (2008).

[41] Karasik, B. S. et al., "Electrical NEP in hot-electron titanium superconducting bolometers," in Millimeter and Submillimeter Detectors and Instrumentation for Astronomy IV, W.D. Duncan, W.S. Holland, S. Withington \& J. Zmuidzinas, eds., Proc. SPIE, 7020, 70200E-70200E-8 (2008).

[42] Mazin, B.A. et al., "Superconducting Kinetic Inductance Photon Detectors," in Highly Innovative Space Telescope Concepts, H.A. MacEwen, ed., Proc. SPIE, 4849, 283 (2002).

[43] Sergeev, A., Karasik, B., Gogidze, I., and Mitin, V., "Ultrasensitive Hot-Electron Kinetic-Inductance Detectors," in Ninth International Workshop On Low Temperature Detectors, F.S. Porter, D. McCammon, M. Galeazzi \& C.K. Stahle, eds., AIP Conf. Proc., 605, 27 (2002).

[44] Baselmans, J. et al., "Development of high-Q superconducting resonators for use as kinetic inductance detectors," Adv. Sp. Res., 40, 708 (2007).

[45]DiPirro, M. et al., "High-fidelity cryothermal test of a subscale large space telescope," in Cryogenic Optical Systems and Instruments XII, J.B. Heaney \& L.G. Burriesci, eds., Proc. SPIE, 6692, 669202 (2007).

[46]Lyon, R.G. et al., "Wide-field imaging interferometry spatial-spectral image synthesis algorithms," in Optical and Infrared Interferometry III, F. Delplancke, J.K. Rajagopal \& F. Malbet, eds., Proc. SPIE 8445 (2012).

[47]Ade, P.A.R., Pisano, G., Tucker, C.E., and Weaver, S.O., "A review of metal mesh filters," in Millimeter and Submillimeter Detectors and Instrumentation for Astronomy III, J. Zmuidzinas, W.S. Holland, S. Withington \& W.D. Duncan, eds., Proc. SPIE, 6275, 62750U (2006).

[48] van den Dool, T.C. et al., "The manufacturing, assembly and acceptance testing of the breadboard cryogenic optical delay line for DARWIN," in Cryogenic Optical Systems and Instruments XI, J.B. Heaney \& L.G. Burriesci, eds., Proc. SPIE, 5904, 590415-1 (2005).

[49] Nemati, B., "External metrology truss technology demonstration (KITE), in Interferometry in Space, M. Shao, ed., Proc. SPIE 4852, 90 (2003).

[50] Lorenzini, E. et al., "Far-Infrared/Submillimeter Astronomical Interferometry with Spaceborne Tether Formations," Ap. \& Sp. Sci., 302, 225 (2006).

[51] Chung, S.-J. et al., "SPHERES Tethered Formation Flight Testbed: Advancements in Enabling NASA's SPECS Mission," in Advances in Stellar Interferometry, J.D. Monnier, M. Schöller \& W.C. Danchi, eds., Proc. SPIE 6268, 62680B (2006).

[52]Nakagawa, T., Matsuhara, H., and Kawakatsu, Y., "The next-generation infrared space telescope SPICA," in Space Telescopes and Instrumentation 2012: Optical, Infrared, and Millimeter Wave, M.C. Clampin, G.G. Fazio, H.A. MacEwen \& J.M. Oschmann, Jr., eds., Proc. SPIE, 8442 (this volume) (2012).

[53]Bolcar, M.R., Leisawitz, D.T., Maher, S.F., and Rinehart, S.A., "Demonstration of the wide-field imaging interferometer testbed using a calibrated hyperspectral image projector," in Optical and Infrared Interferometry III, F. Delplancke, J.K. Rajagopal \& F. Malbet, eds., Proc. SPIE, 8445 (2012).

[54]Rinehart, S.A. et al., "Design and status of the Balloon Experimental Twin Telescope for infrared interferometry (BETTII): an interferometer at the edge of space," in Optical and Infrared Interferometry III, F. Delplancke, J.K. Rajagopal, \& F. Malbet, eds., Proc. SPIE, 8445 (2012).

[55] Shibai, H. et al., "Far-Infrared Interferometric Experiment (FITE): Toward the First Flight," in Pathways Towards Habitable Planets, V. Coudé du Foresto, D.M. Gelino \& I. Ribas, eds., San Francisco: Astronomical Society of the Pacific, p. 541 (2010).

[56] Caldwell, M.E., Swinyard, B.M., and Ferlet, M.J., "Test-bed interferometer to develop active optics techniques for space interferometers in mid-IR and far-IR astronomy missions," in Joint 32nd International Conf. on IR and Millimeter Waves, 2007, and the 2007 15th International Conference on Teraherts Electronics, IRMMW-THz, p. 182 (2007). 